\title{
Existence Results for a Coupled System of Nonlinear Fourth-Order Differential Equations
}

\author{
Bessem Samet \\ Department of Mathematics, College of Science, King Saud University, P.O. Box 2455, Riyadh 11451, Saudi Arabia \\ Correspondence should be addressed to Bessem Samet; bessem.samet@gmail.com
}

Received 10 November 2013; Accepted 3 December 2013

Academic Editor: Mohamed Boussairi Jleli

Copyright (C) 2013 Bessem Samet. This is an open access article distributed under the Creative Commons Attribution License, which permits unrestricted use, distribution, and reproduction in any medium, provided the original work is properly cited.

Sufficient conditions are obtained for the existence of solutions to a coupled system of nonlinear fourth-order differential equations.

\section{Introduction}

The purpose of this paper is to study the existence of solutions to the following coupled system of nonlinear fourth-order differential equations:

$$
\begin{gathered}
x^{\prime \prime \prime \prime}(t)=f(t, x(t), y(t)), \quad t \in(0,1), \\
y^{\prime \prime \prime \prime}(t)=g(t, x(t), y(t)), \quad t \in(0,1), \\
x(0)=x^{\prime \prime}(0)=0, \quad x^{\prime}(1)=0, \\
x^{\prime \prime \prime}(1)=\xi(x(1)), \\
y(0)=y^{\prime \prime}(0)=0, \quad y^{\prime}(1)=0, \\
y^{\prime \prime \prime}(1)=\zeta(y(1)),
\end{gathered}
$$

where $f, g:[0,1] \times \mathbb{R} \times \mathbb{R} \rightarrow \mathbb{R}$ and $\xi, \zeta: \mathbb{R} \rightarrow \mathbb{R}$ are given continuous functions.

In recent years, boundary value problems for fourthorder ordinary differential equations have been extensively studied. Gupta [1] considered the boundary value problem

$$
\begin{gathered}
x^{\prime \prime \prime \prime}(t)+f(t) x(t)=g(t), \quad t \in(0, \pi), \\
x^{\prime}(0)=x^{\prime \prime \prime}(0)=x^{\prime \prime \prime}(\pi)=x^{\prime}(\pi)=0,
\end{gathered}
$$

which describes the unstable static equilibrium of an elastic beam which is supported by sliding clamps at both ends, $t=0$ and $t=\pi$. Many authors (see [2-8] and references therein) studied the existence of solutions to the problem

$$
\begin{gathered}
x^{\prime \prime \prime \prime}(t)=f\left(t, x(t), x^{\prime \prime}(t)\right), \quad t \in(0,1), \\
x(0)=x(1)=x^{\prime \prime}(0)=x^{\prime \prime}(1)=0,
\end{gathered}
$$

which describes the deformation of the equilibrium state of an elastic beam with its two ends being simply supported. For other results on the study of fourth-order ordinary differential equations, we refer to [9-13] and references therein. On the other hand, to our knowledge, there are only few papers concerning existence results for systems of fourthorder ordinary differential equations. For example, in [14], via the fixed point index theory, the authors studied the existence of solutions to the system of bending elastic beam equations

$$
\begin{aligned}
x^{\prime \prime \prime \prime}(t)= & f\left(t, x(t), y(t), x^{\prime \prime}(t), y^{\prime \prime}(t)\right), \quad t \in(0,1), \\
y^{\prime \prime \prime \prime}(t)= & g\left(t, x(t), y(t), x^{\prime \prime}(t), y^{\prime \prime}(t)\right), \quad t \in(0,1), \\
& x(0)=x(1)=x^{\prime \prime}(0)=x^{\prime \prime}(1)=0, \\
& y(0)=y(1)=y^{\prime \prime}(0)=y^{\prime \prime}(1)=0 .
\end{aligned}
$$

In this work, we provide four sufficient conditions for the existence of solutions to the problem (1). Some illustrative examples are presented.

The paper is organized as follows. In Section 2, we present the main results. In Section 3, we give some illustrative 
examples. In Section 4, we present some basic lemmas that will be used for the proofs. Finally, in Section 5, we prove our results.

\section{Main Results}

We obtained the following existence results.

Theorem 1. Suppose the following conditions hold:

(i) there exist $\alpha, \beta, \lambda, \mu>0$ such that, for all $t \in[0,1]$ and $p, q, r, e \in \mathbb{R}$, one has

$$
\begin{gathered}
|f(t, p, q)-f(t, r, e)| \leq \alpha|p-r|+\beta|q-e|, \\
|g(t, p, q)-g(t, r, e)| \leq \lambda|p-r|+\mu|q-e|,
\end{gathered}
$$

(ii) there exist $\gamma, \theta>0$ such that, for all $p, q \in \mathbb{R}$, one has

$$
\begin{gathered}
|\xi(p)-\xi(q)| \leq \gamma|p-q|, \\
|\zeta(p)-\zeta(q)| \leq \theta|p-q|,
\end{gathered}
$$

(iii) $\rho(A)<1$, where $A \in M_{2,2}\left(\mathbb{R}_{+}\right)$is the matrix given by

$$
A=\frac{1}{2}\left(\begin{array}{ll}
\gamma+\alpha & \beta \\
\theta+\lambda & \mu
\end{array}\right)
$$

and $\rho(A)$ denotes the spectral radius of $A$. Then

(I) equation (1) has a unique solution $\left(x^{*}, y^{*}\right) \in C([0,1]$; $\mathbb{R}) \times C([0,1] ; \mathbb{R})$,

(II) for any $u_{0}, v_{0} \in C([0,1] ; \mathbb{R})$, the sequences $\left\{u_{n}\right\},\left\{v_{n}\right\} \subset$ $C([0,1] ; \mathbb{R})$ defined by

$$
u_{n+1}=F_{1}\left(u_{n}, v_{n}\right), \quad v_{n+1}=F_{2}\left(u_{n}, v_{n}\right)
$$

converge, respectively, to $x^{*}$ and $y^{*}$; that is,

$$
\lim _{n \rightarrow \infty}\left\|u_{n}-x^{*}\right\|_{\infty}=\lim _{n \rightarrow \infty}\left\|v_{n}-y^{*}\right\|_{\infty}=0 .
$$

Theorem 2. Suppose the following conditions hold:

(i) there exists a nonnegative function $a \in L^{1}(0,1)$ such that, for all $t \in[0,1]$ and $p, q \in \mathbb{R}$, one has

$$
\begin{array}{r}
|f(t, p, q)| \leq a(t)+\epsilon_{1}|p|^{\rho_{1}}+\epsilon_{2}|q|^{\rho_{2}}, \\
\epsilon_{1}, \epsilon_{2}>0, \quad 0<\rho_{1}, \quad \rho_{2}<1,
\end{array}
$$

(ii) there exists a nonnegative function $b \in L^{1}(0,1)$ such that, for all $t \in[0,1]$ and $p, q \in \mathbb{R}$, one has

$$
\begin{array}{r}
|g(t, p, q)| \leq b(t)+\delta_{1}|p|^{\sigma_{1}}+\delta_{2}|q|^{\sigma_{2}}, \\
\delta_{1}, \delta_{2}>0, \quad 0<\sigma_{1}, \quad \sigma_{2}<1,
\end{array}
$$

(iii) for all $p \in \mathbb{R}$, one has

$$
\max \{|\xi(p)|,|\zeta(p)|\} \leq|p|
$$

Then (1) has at least one solution $\left(x^{*}, y^{*}\right) \in C([0,1] ; \mathbb{R}) \times$ $C([0,1] ; \mathbb{R})$.

Theorem 3. Suppose the following conditions hold:

(i) for all $t \in[0,1]$ and $p, q \in \mathbb{R}$, one has

$$
|f(t, p, q)| \leq a+\epsilon|p|, \quad a>0,0 \leq \epsilon<1,
$$

(ii) for all $t \in[0,1]$ and $p, q \in \mathbb{R}$, one has

$$
|g(t, p, q)| \leq b+\delta|q|, \quad b>0,0 \leq \delta<1,
$$

(iii) for all $p \in \mathbb{R}$, one has

$$
\max \{|\xi(p)|,|\zeta(p)|\} \leq|p| .
$$

Then (1) has at least one solution $\left(x^{*}, y^{*}\right) \in C([0,1] ; \mathbb{R}) \times$ $C([0,1] ; \mathbb{R})$.

Theorem 4. Suppose the following conditions hold:

(i) for all $t \in[0,1]$ and $p, q \in \mathbb{R}$, one has

$$
\begin{aligned}
& |f(t, p, q)| \leq a+\epsilon_{1}|p|^{\rho_{1}}+\epsilon_{2}|q|^{\rho_{2}}, \\
& 0 \leq 2 a<1, \quad \epsilon_{1}, \epsilon_{2}>0, \quad 2\left(\epsilon_{1}+\epsilon_{2}\right) \leq 1, \quad \rho_{1}, \rho_{2} \geq 1,
\end{aligned}
$$

(ii) for all $t \in[0,1]$ and $p, q \in \mathbb{R}$, one has

$$
\begin{aligned}
& |g(t, p, q)| \leq b+\delta_{1}|p|^{\sigma_{1}}+\delta_{2}|q|^{\sigma_{2}}, \\
& 0 \leq 2 b<1, \quad \delta_{1}, \delta_{2}>0, \quad 2\left(\delta_{1}+\delta_{2}\right) \leq 1, \quad \sigma_{1}, \sigma_{2} \geq 1,
\end{aligned}
$$

(iii) for all $p, q \in \mathbb{R}$, one has

$$
\begin{array}{r}
\max \{|\xi(p)-\xi(q)|,|\zeta(p)-\zeta(q)|\} \leq k|p-q| \\
0<k<2
\end{array}
$$

(iv) $\xi(0)=\zeta(0)=0$.

Then (1) has at least one solution $\left(x^{*}, y^{*}\right) \in C([0,1] ; \mathbb{R}) \times$ $C([0,1] ; \mathbb{R})$.

\section{Illustrative Examples}

Example 1. Consider the initial value problem

$$
x^{\prime \prime \prime \prime}(t)=\left(\frac{1}{2} \sin (x(t))+\frac{3 \sqrt{3}}{16} \sin (y(t))\right) \cos (t),
$$

$$
\begin{gathered}
y^{\prime \prime \prime \prime}(t)=\left(\frac{4}{3}|x(t)|+\frac{\sqrt{3}}{2}|y(t)|\right) e^{-t} \sin (t), \quad t \in(0,1), \\
x(0)=x^{\prime \prime}(0)=0, \quad x^{\prime}(1)=0,
\end{gathered}
$$




$$
\begin{gathered}
4 x^{\prime \prime \prime}(1)=\sin (2 x(1)), \\
y(0)=y^{\prime \prime}(0)=0, \quad y^{\prime}(1)=0, \\
y^{\prime \prime \prime}(1)=\frac{4}{9} \sin (3(y(1))) .
\end{gathered}
$$

In this case, for all $t \in[0,1]$ and $p, q \in \mathbb{R}$, we have

$$
\begin{gathered}
f(t, p, q)=\left(\frac{1}{2} \sin (p)+\frac{3 \sqrt{3}}{16} \sin (q)\right) \cos (t), \\
g(t, p, q)=\left(\frac{4}{3}|p|+\frac{\sqrt{3}}{2}|q|\right) e^{-t} \sin (t), \\
\xi(p)=\frac{1}{4} \sin (2 p), \\
\zeta(p)=\frac{4}{9} \sin (3 p) .
\end{gathered}
$$

Conditions (i) and (ii) of Theorem 1 are satisfied with

$$
\begin{array}{lll}
\alpha=\frac{1}{2}, & \beta=\frac{3 \sqrt{3}}{16}, & \lambda=\frac{4}{3}, \\
\mu=\frac{\sqrt{3}}{2}, & \gamma=\frac{1}{2}, & \theta=\frac{4}{3} .
\end{array}
$$

Moreover, the matrix

$$
A=\left(\begin{array}{cc}
\frac{\alpha+\gamma}{2} & \frac{\beta}{2} \\
\frac{\lambda+\theta}{2} & \frac{\mu}{2}
\end{array}\right)=\left(\begin{array}{cc}
\frac{1}{2} & \frac{3 \sqrt{3}}{32} \\
\frac{4}{3} & \frac{\sqrt{3}}{4}
\end{array}\right)
$$

has the eigenvalues $\lambda_{1}=0$ and $\lambda_{2}=0.9330$. From Theorem 1 , the considered problem has a unique solution $\left(x^{*}, y^{*}\right) \in$ $C([0,1] ; \mathbb{R}) \times C([0,1] ; \mathbb{R})$.

Example 2. Consider the initial value problem

$$
\begin{gathered}
x^{\prime \prime \prime \prime}(t)=e^{t}+\sin (\sqrt{|x(t)+y(t)|}) \cos (t), \quad t \in(0,1), \\
y^{\prime \prime \prime \prime}(t)=3 t^{2}-5+e^{-t}|x(t)|^{2 / 5}+\frac{\sqrt{|y(t)|}}{2(1+\sqrt{|y(t)|})}, t \in(0,1), \\
x(0)=x^{\prime \prime}(0)=0, \quad x^{\prime}(1)=0, \\
x^{\prime \prime \prime}(1)=\sin (x(1)), \\
y(0)=y^{\prime \prime}(0)=0, \quad y^{\prime}(1)=0, \\
y^{\prime \prime \prime}(1)=|y(1)| .
\end{gathered}
$$

In this case, for all $t \in[0,1]$ and $p, q \in \mathbb{R}$, we have

$$
\begin{gathered}
f(t, p, q)=e^{t}+\sin (\sqrt{|p+q|}) \cos (t), \\
g(t, p, q)=3 t^{2}-5+e^{-t}|p|^{2 / 5}+\frac{\sqrt{|q|}}{2(1+\sqrt{|q|})}, \\
\xi(p)=\sin (p), \\
\zeta(p)=|p| .
\end{gathered}
$$

Conditions (i) and (ii) of Theorem 2 are satisfied with

$$
\begin{gathered}
a(t)=e^{t}, \quad \epsilon_{1}=\epsilon_{2}=1, \quad \rho_{1}=\rho_{2}=\frac{1}{2}, \\
b(t)=3 t^{2}-5, \quad \delta_{1}=1, \\
\delta_{2}=\frac{1}{2}, \quad \sigma_{1}=\frac{2}{5}, \quad \sigma_{2}=\frac{1}{2} .
\end{gathered}
$$

From Theorem 2, the considered problem has at least one solution $\left(x^{*}, y^{*}\right) \in C([0,1] ; \mathbb{R}) \times C([0,1] ; \mathbb{R})$.

Example 3. Consider the initial value problem

$$
\begin{gathered}
x^{\prime \prime \prime \prime}(t)=e^{-t}+\frac{1}{2} \sin \left(\frac{x(t)}{1+y^{2}(t)}\right), \quad t \in(0,1), \\
y^{\prime \prime \prime \prime}(t)=\frac{1}{1+t^{2}+x^{2}(t)+y^{2}(t)}, \quad t \in(0,1), \\
x(0)=x^{\prime \prime}(0)=0, \quad x^{\prime}(1)=0, \\
x^{\prime \prime \prime}(1)=\sin \left(\frac{x(1)}{1+x(1)^{2}}\right) \\
y(0)=y^{\prime \prime}(0)=0, \quad y^{\prime}(1)=0 \\
y^{\prime \prime \prime}(1)=\frac{y(1)}{1+y(1)^{4}} .
\end{gathered}
$$

In this case, for all $t \in[0,1]$ and $p, q \in \mathbb{R}$, we have

$$
\begin{gathered}
f(t, p, q)=e^{-t}+\frac{1}{2} \sin \left(\frac{p}{1+q^{2}}\right) \\
g(t, p, q)=\frac{1}{1+t^{2}+p^{2}+q^{2}} \\
\xi(p)=\sin \left(\frac{p}{1+p^{2}}\right) \\
\zeta(p)=\frac{p}{1+p^{4}}
\end{gathered}
$$

Conditions (i) and (ii) of Theorem 3 are satisfied with

$$
a=1, \quad \epsilon=\frac{1}{2}, \quad b=1, \quad \delta=0 .
$$

From Theorem 3, the considered problem has at least one solution $\left(x^{*}, y^{*}\right) \in C([0,1] ; \mathbb{R}) \times C([0,1] ; \mathbb{R})$. 
Example 4. Consider the initial value problem

$$
\begin{gathered}
x^{\prime \prime \prime \prime}(t)=\frac{t^{2}}{5}+\frac{x(t)^{2}+y(t)^{2}}{4} \cos (t), \quad t \in(0,1), \\
y^{\prime \prime \prime \prime}(t)=\frac{t e^{t-1}}{3}+\frac{\sin (x(t)+y(t))}{4}, \quad t \in(0,1), \\
x(0)=x^{\prime \prime}(0)=0, \quad x^{\prime}(1)=0 \\
x^{\prime \prime \prime}(1)=\sin \left(\frac{3 x(1)}{2}\right) \\
y(0)=y^{\prime \prime}(0)=0, \quad y^{\prime}(1)=0 \\
y^{\prime \prime \prime}(1)=\frac{3}{2}|y(1)| .
\end{gathered}
$$

In this case, for all $t \in[0,1]$ and $p, q \in \mathbb{R}$, we have

$$
\begin{gathered}
f(t, p, q)=\frac{t^{2}}{5}+\frac{p^{2}+q^{2}}{4} \cos (t), \\
g(t, p, q)=\frac{t e^{t-1}}{3}+\frac{\sin (p+q)}{4}, \\
\xi(p)=\sin \left(\frac{3 p}{2}\right), \\
\zeta(p)=\frac{3|p|}{2} .
\end{gathered}
$$

Conditions (i), (ii), and (iii) of Theorem 4 are satisfied with

$$
\begin{gathered}
a=\frac{1}{5}, \quad \epsilon_{1}=\epsilon_{2}=\frac{1}{4}, \quad \rho_{1}=\rho_{2}=2, \\
b=\frac{1}{3}, \quad \delta_{1}=1, \quad \delta_{2}=\frac{1}{4}, \\
\sigma_{1}=\sigma_{2}=1, \quad k=\frac{3}{2} .
\end{gathered}
$$

From Theorem 4, the considered problem has at least one solution $\left(x^{*}, y^{*}\right) \in C([0,1] ; \mathbb{R}) \times C([0,1] ; \mathbb{R})$.

\section{Preliminaries}

In order to obtain an integral formulation of the problem (1), we need the following lemma. At first, we denote by $C([0,1] ; \mathbb{R})$ the set of continuous functions from $[0,1]$ into $\mathbb{R}$.

Lemma 5. Let $\rho \in C([0,1] ; \mathbb{R})$ and $\omega \in \mathbb{R}$. The problem

$$
\begin{gathered}
x^{\prime \prime \prime \prime}(t)=\rho(t), \quad t \in(0,1), \\
x(0)=x^{\prime \prime}(0)=0, \quad x^{\prime}(1)=0, \quad x^{\prime \prime \prime}(1)=\omega
\end{gathered}
$$

has a unique solution

$$
x(t)=\int_{0}^{1} G(t, s) \rho(s) d s+\varphi_{\omega}(t),
$$

where

$$
\begin{gathered}
\varphi_{\omega}(t)=\frac{\omega}{2} t\left(\frac{t^{2}}{3}-1\right), \\
G(t, s)=\frac{1}{6} \begin{cases}t\left(6 s-t^{2}-3 s^{2}\right), & 0 \leq t \leq s, \\
s\left(6 t-s^{2}-3 t^{2}\right), & s \leq t \leq 1 .\end{cases}
\end{gathered}
$$

Proof. We omit the proof as it employs standard arguments.

From Lemma 5, problem (1) is equivalent to the following system of integral equations:

$$
\begin{array}{r}
x(t)=\int_{0}^{1} G(t, s) f(s, x(s), y(s)) d s+\varphi_{\xi(x(1))}(t), \\
y(t)=\int_{0}^{1} G(t, s) g(s, x(s), y(s)) d s+\varphi_{\zeta(y(1))}(t), \\
t \in[0,1] .
\end{array}
$$

Let $X=C([0,1] ; \mathbb{R})$. Define the mappings $F_{1}, F_{2}: X \times X \rightarrow$ $X$ by

$$
\begin{gathered}
F_{1}(u, v)(t)=\int_{0}^{1} G(t, s) f(s, u(s), v(s)) d s+\varphi_{\xi(u(1))}(t), \\
F_{2}(u, v)(t)=\int_{0}^{1} G(t, s) g(s, u(s), v(s)) d s+\varphi_{\zeta(v(1))}(t)
\end{gathered}
$$

for every $t \in[0,1]$. Let $F: X \times X \rightarrow X \times X$ be the mapping defined by

$$
F(u, v)=\left(F_{1}(u, v), F_{2}(u, v)\right) .
$$

Observe that $(x, y) \in X \times X$ is a solution to (35) if and only if $(x, y)$ is a fixed point of $F$.

Lemma 6. One has

$$
0 \leq \frac{t^{2} s}{3} \leq G(t, s) \leq \frac{1}{2} s\left(2 t-t^{2}\right) \leq \frac{1}{2}, \quad \forall t, s \in[0,1]
$$

Proof. For $0 \leq t \leq s \leq 1$, we have

$$
\frac{\partial G(t, s)}{\partial t}=(1-t) s-\frac{1}{2}(s-t)^{2} \leq(1-t) s .
$$

Similarly, for $s \leq t \leq 1$, we have

$$
\frac{\partial G(t, s)}{\partial t}=(1-t) s .
$$

Then, for all $t, s \in[0,1]$, we have

$$
\begin{aligned}
G(t, s) & =\int_{0}^{t} \frac{\partial G(r, s)}{\partial r} d r \\
& \leq \int_{0}^{t}(1-r) s d r=\frac{1}{2} s\left(2 t-t^{2}\right) \leq \frac{1}{2} .
\end{aligned}
$$


Now, for $0 \leq t \leq s \leq 1$, we have

$$
G(t, s)=\frac{t}{6}\left(6 s-t^{2}-3 s^{2}\right) \geq \frac{t}{6}\left(6 s-4 s^{2}\right) \geq \frac{t^{2} s}{3} .
$$

Similarly, for $s \leq t \leq 1$, we have

$$
G(t, s)=\frac{s}{6}\left(6 t-s^{2}-3 t^{2}\right) \geq \frac{s}{6}\left(6 t-4 t^{2}\right) \geq \frac{t^{2} s}{3} .
$$

We endow the product set $Z=X \times X$ with the norm

$$
\|(x, y)\|_{Z}=\max \left\{\|x\|_{\infty},\|y\|_{\infty}\right\}
$$

where

$$
\|x\|_{\infty}=\max \{|x(t)|: t \in[0,1]\} .
$$

Lemma 7. The mapping $F: Z \rightarrow Z$ is continuous with respect to the norm $\|\cdot\|_{Z}$.

Proof. Let $(x, y) \in Z$ be an arbitrary point. Let $R$ be a nonnegative real number such that $\|(x, y)\|_{\mathrm{Z}} \leq R$. To show the continuity of $F$ at the point $(x, y)$, let us consider a sequence $\left\{\left(x_{n}, y_{n}\right)\right\} \subset Z$ converging to $(x, y)$; that is,

$$
\lim _{n \rightarrow \infty}\left\|x_{n}-x\right\|_{\infty}=\lim _{n \rightarrow \infty}\left\|y_{n}-y\right\|_{\infty}=0 .
$$

Without restriction to the generality, we can suppose that $\left\|\left(x_{n}, y_{n}\right)\right\|_{Z} \leq R$, for every $n \in \mathbb{N}$ ( $\mathbb{N}$ is the set of natural numbers). Since $f$ is continuous, we can define

$$
M=\max \{|f(t, p, q)|: 0 \leq t \leq 1,-R \leq p, q \leq R\} .
$$

For all $t \in[0,1]$, we have

$$
\begin{aligned}
& \left|F_{1}\left(x_{n}, y_{n}\right)(t)-F_{1}(x, y)(t)\right| \\
& \leq \int_{0}^{1} G(t, s)\left|f\left(s, x_{n}(s), y_{n}(s)\right)-f(s, x(s), y(s))\right| d s \\
& \quad+\frac{1}{2}\left|\xi\left(x_{n}(1)\right)-\xi(x(1))\right| .
\end{aligned}
$$

Using Lemma 6 , for all $t \in[0,1]$, we have

$$
\begin{aligned}
& 2\left|F_{1}\left(x_{n}, y_{n}\right)(t)-F_{1}(x, y)(t)\right| \\
& \leq \int_{0}^{1}\left|f\left(s, x_{n}(s), y_{n}(s)\right)-f(s, x(s), y(s))\right| d s \\
& \quad+\left|\xi\left(x_{n}(1)\right)-\xi(x(1))\right|,
\end{aligned}
$$

which implies that

$$
\begin{aligned}
& 2\left\|F_{1}\left(x_{n}, y_{n}\right)-F_{1}(x, y)\right\|_{\infty} \\
& \leq \int_{0}^{1}\left|f\left(s, x_{n}(s), y_{n}(s)\right)-f(s, x(s), y(s))\right| d s \\
& \quad+\left|\xi\left(x_{n}(1)\right)-\xi(x(1))\right| .
\end{aligned}
$$

From the continuity of $\xi$, we have

$$
\lim _{n \rightarrow \infty}\left|\xi\left(x_{n}(1)\right)-\xi(x(1))\right|=0 .
$$

The continuity of $f$ implies that, for all $s \in[0,1]$,

$$
\lim _{n \rightarrow \infty}\left|f\left(s, x_{n}(s), y_{n}(s)\right)-f(s, x(s), y(s))\right|=0 .
$$

On the other hand, for all $n \in \mathbb{N}$, we have

$$
\int_{0}^{1}\left|f\left(s, x_{n}(s), y_{n}(s)\right)-f(s, x(s), y(s))\right| d s \leq 2 M .
$$

Then, by the dominated convergence theorem, we have

$$
\lim _{n \rightarrow \infty} \int_{0}^{1}\left|f\left(s, x_{n}(s), y_{n}(s)\right)-f(s, x(s), y(s))\right| d s=0 .
$$

Consequently, we have

$$
\lim _{n \rightarrow \infty}\left\|F_{1}\left(x_{n}, y_{n}\right)-F_{1}(x, y)\right\|_{\infty}=0 .
$$

Similarly, we can show that

$$
\lim _{n \rightarrow \infty}\left\|F_{2}\left(x_{n}, y_{n}\right)-F_{2}(x, y)\right\|_{\infty}=0
$$

We deduce that

$$
\lim _{n \rightarrow \infty}\left\|F\left(x_{n}, y_{n}\right)-F(x, y)\right\|_{Z}=0,
$$

which yields the desired result.

Lemma 8. Let $D$ be the subset of $Z$ defined by

$$
D=\left\{(x, y) \in Z:\|(x, y)\|_{Z} \leq R\right\},
$$

for some $R>0$. Under condition (iii) of Theorem 2 (or conditions (iii)-(iv) of Theorem 4), $F(D)$ is an equicontinuous set.

Proof. Suppose that (iii) of Theorem 2 holds. Since $f$ and $g$ are continuous, we can define

$$
\begin{aligned}
& M=\max \{|f(t, p, q)|: 0 \leq t \leq 1,-R \leq p, q \leq R\}, \\
& N=\max \{|g(t, p, q)|: 0 \leq t \leq 1,-R \leq p, q \leq R\} .
\end{aligned}
$$

Let $\varepsilon>0$. Since $G$ is continuous in $[0,1] \times[0,1]$, there exists $\alpha>0$ such that

$$
\left|G(t, s)-G\left(t^{\prime}, s\right)\right|<\min \left\{\frac{\varepsilon}{2 M}, \frac{\varepsilon}{2 N}\right\}
$$


whenever $\left|t-t^{\prime}\right|<\alpha$. Let $\kappa=\min \{\alpha, \varepsilon / 2 R\}$. For $(x, y) \in D$, for $\left|t-t^{\prime}\right|<\kappa$, we have

$$
\begin{aligned}
\mid & F_{1}(x, y)(t)-F_{1}(x, y)\left(t^{\prime}\right) \mid \\
\leq & \int_{0}^{1}\left|G(t, s)-G\left(t^{\prime}, s\right)\right||f(s, x(s), y(s))| d s \\
& +|\xi(x(1))|\left|t-t^{\prime}\right| \\
\leq & M \frac{\varepsilon}{2 M}+R \frac{\varepsilon}{2 R} \text { (from (iii) of Theorem 2) } \\
= & \varepsilon \\
\mid & F_{2}(x, y)(t)-F_{2}(x, y)\left(t^{\prime}\right) \mid \\
\leq & \int_{0}^{1}\left|G(t, s)-G\left(t^{\prime}, s\right)\right||g(s, x(s), y(s))| d s \\
& +|\zeta(y(1))|\left|t-t^{\prime}\right| \\
\leq & N \frac{\varepsilon}{2 N}+R \frac{\varepsilon}{2 R} \text { (from (iii) of Theorem 2) } \\
= & \varepsilon .
\end{aligned}
$$

Then, the equicontinuity of $F(D)$ is proved. If conditions (iii)(iv) of Theorem 4 hold, using the same technique with $\kappa=$ $\min \{\alpha, \varepsilon / 2 k R\}$, we get the desired result.

Now, we are ready to prove our main results.

\section{The Proofs}

5.1. Proof of Theorem 1. The proof is based on Perov fixed point theorem [15]. At first, let us recall some concepts.

Let $m \geq 1$ be a positive integer. We endow the set $\mathbb{R}^{m}$ with the partial order $\unlhd_{m}$ defined by

$$
\begin{array}{r}
\alpha=\left(\alpha_{1}, \ldots, \alpha_{m}\right), \quad \beta=\left(\beta_{1}, \ldots, \beta_{m}\right) \in \mathbb{R}^{m}, \\
\alpha \unlhd_{m} \beta \Longleftrightarrow \alpha_{i} \leq \beta_{i}, \quad \forall i=1, \ldots, m .
\end{array}
$$

We denote by $0_{\mathbb{R}^{m}}$ the zero vector of $\mathbb{R}^{\mathrm{m}}$.

Let $\Sigma$ be a nonempty set. A mapping $d: \Sigma \times \Sigma \rightarrow R^{m}$ is called a vector valued metric on $\Sigma$ if the following properties are satisfied:

(d1) $d(x, y)=0_{\mathbb{R}^{m}} \Leftrightarrow x=y$, for all $x, y \in \Sigma ;$

(d2) $d(x, y) \geq_{m} 0_{\mathbb{R}^{m}}$, for all $x, y \in \Sigma$;

(d3) $d(x, y)=d(y, x)$, for all $x, y \in \Sigma$;

(d4) $d(x, y) \unlhd_{m} d(x, z)+d(z, y)$, for all $x, y, z \in \Sigma$.

A set $\Sigma$ equipped with a vector valued metric $d$ is called a generalized metric space. We will denote such a space by $(\Sigma, d)$. For the generalized metric spaces, the notions of convergent sequence, Cauchy sequence, completeness, open subset, and closed subset are similar to those for usual metric spaces.

We denote by $M_{m, m}\left(\mathbb{R}_{+}\right)$the set of all $m \times m$ matrices with positive elements. For $A \in M_{m, m}\left(\mathbb{R}_{+}\right)$, we denote by $\rho(A)$ the spectral radius of $A$, that is, the supremum among the absolute values of the elements in its spectrum.

Lemma 9. Let $(M, d)$ be a complete generalized metric space and the mapping $T: M \rightarrow M$ with the property that there exists a matrix $A \in M_{m, m}\left(\mathbb{R}_{+}\right)$such that

$$
d(T x, T y) \unlhd_{m} A d(x, y), \quad \forall x, y \in M .
$$

If $\rho(A)<1$, then $T$ has a unique fixed point $x^{*} \in M$. Moreover, for any $x_{0} \in M$, the iterative sequence $\left\{x_{n}\right\} \subset M$ defined by $x_{n+1}=T x_{n}$ converges to $x^{*}$.

Proof. We endow $Z=X \times X$ with the vector valued metric $d: Z \times Z \rightarrow \mathbb{R}^{2}$ defined by

$$
d((x, y),(u, v))=\left(\begin{array}{l}
\|x-u\|_{\infty} \\
\|y-v\|_{\infty}
\end{array}\right), \quad \forall(x, y),(u, v) \in Z .
$$

It is easy to show that $(Z, d)$ is a complete generalized metric space. Let $(x, y),(u, v) \in Z$. For any $t \in[0,1]$, we have

$$
\begin{aligned}
& F_{1}(x, y)(t)-F_{1}(u, v)(t) \\
&= \int_{0}^{1} G(t, s) f(s, x(s), y(s))-f(s, u(s), v(s)) d s \\
&+\varphi_{\xi(x(1))}(t)-\varphi_{\xi(u(1))}(t) \\
&= \int_{0}^{1} G(t, s) f(s, x(s), y(s))-f(s, u(s), v(s)) d s \\
&+t\left(\frac{t^{2}}{3}-1\right) \frac{\xi(x(1))-\xi(u(1))}{2} .
\end{aligned}
$$

Using Lemma 6, for all $t \in[0,1]$, we have

$$
\begin{aligned}
& 2\left|F_{1}(x, y)(t)-F_{1}(u, v)(t)\right| \\
& \leq \int_{0}^{1}|f(s, x(s), y(s))-f s, u(s), v(s)| d s \\
& \quad+|\xi(x(1))-\xi(u(1))| .
\end{aligned}
$$

Using conditions (i) and (ii), we get that

$$
\begin{aligned}
\left|F_{1}(x, y)(t)-F_{1}(u, v)(t)\right| \leq & \left(\frac{\gamma+\alpha}{2}\right)\|x-u\|_{\infty} \\
& +\left(\frac{\beta}{2}\right)\|y-v\|_{\infty},
\end{aligned}
$$

for all $t \in[0,1]$. This implies that

$$
\begin{aligned}
\left\|F_{1}(x, y)-F_{1}(u, v)\right\|_{\infty} \leq & \left(\frac{\gamma+\alpha}{2}\right)\|x-u\|_{\infty} \\
& +\left(\frac{\beta}{2}\right)\|y-v\|_{\infty} .
\end{aligned}
$$

Using similar calculations, we obtain that

$$
\begin{aligned}
& \left\|F_{2}(x, y)-F_{2}(u, v)\right\|_{\infty} \\
& \quad \leq\left(\frac{\theta+\lambda}{2}\right)\|x-u\|_{\infty}+\left(\frac{\mu}{2}\right)\|y-v\|_{\infty} .
\end{aligned}
$$


It follows from (68) and (69) that

$$
\begin{array}{r}
d(F(x, y), F(u, v)) \unlhd_{2} A d((x, y),(u, v)), \\
\forall(x, y),(u, v) \in Z,
\end{array}
$$

where

$$
A=\frac{1}{2}\left(\begin{array}{ll}
\gamma+\alpha & \beta \\
\theta+\lambda & \mu
\end{array}\right)
$$

Since $\rho(A)<1$, the desired result follows from Lemma 9 .

5.2. Proof of Theorem 2. The proof is based on Schauder fixed point theorem [16].

Lemma 10. Let $D$ be a closed bounded convex subset of a normed space $E$. If $T: D \rightarrow E$ is a continuous compact map such that $T(D) \subseteq D$, then $T$ has a fixed point.

Proof. Let $D \subset Z$ be the set defined by

$$
D=\left\{(x, y) \in Z:\|(x, y)\|_{Z} \leq R\right\},
$$

where

$$
\begin{array}{r}
R \geq \max \left\{3 \int_{0}^{1} a(s) d s, 3 \int_{0}^{1} b(s) d s,\left(3 \epsilon_{1}\right)^{1 /\left(1-\rho_{1}\right)},\right. \\
\left.\left(3 \epsilon_{2}\right)^{1 /\left(1-\rho_{2}\right)},\left(3 \delta_{1}\right)^{1 /\left(1-\sigma_{1}\right)},\left(3 \delta_{2}\right)^{1 /\left(1-\sigma_{2}\right)}\right\} .
\end{array}
$$

We will prove that

$$
F(D) \subseteq D
$$

Let $(x, y) \in D$; that is, $\|x\|_{\infty} \leq R$ and $\|y\|_{\infty} \leq R$. For all $t \in[0,1]$, we have

$$
F_{1}(x, y)(t)=\int_{0}^{1} G(t, s) f(s, x(s), y(s)) d s+\varphi_{\xi(x(1))}(t) .
$$

Using Lemma 6 and conditions (i) and (iii), we obtain that

$$
\begin{aligned}
\left|F_{1}(x, y)(t)\right| \leq & \frac{1}{2}\left(\int_{0}^{1} a(s) d s\right) \\
& +\frac{\epsilon_{1}}{2} R^{\rho_{1}}+\frac{\epsilon_{2}}{2} R^{\rho_{2}}+\frac{R}{2} \\
\leq & \frac{R}{6}+\frac{R}{6}+\frac{R}{6}+\frac{R}{2}=R .
\end{aligned}
$$

Thus, we have

$$
\left\|F_{1}(x, y)\right\|_{\infty} \leq R
$$

Similarly, for all $t \in[0,1]$, we have

$$
F_{2}(x, y)(t)=\int_{0}^{1} G(t, s) g(s, x(s), y(s)) d s+\varphi_{\zeta(y(1))}(t)
$$

Using Lemma 6 and conditions (ii) and (iii), we obtain that

$$
\begin{aligned}
\left|F_{2}(x, y)(t)\right| \leq & \frac{1}{2}\left(\int_{0}^{1} b(s) d s\right) \\
& +\frac{\delta_{1}}{2} R^{\sigma_{1}}+\frac{\delta_{2}}{2} R^{\sigma_{2}}+\frac{R}{2} \\
\leq & \frac{R}{6}+\frac{R}{6}+\frac{R}{6}+\frac{R}{2}=R .
\end{aligned}
$$

Thus, we have

$$
\left\|F_{2}(x, y)\right\|_{\infty} \leq R \text {. }
$$

Then, (74) follows from (77) and (80).

Now, using Lemmas 7 and 8, Arzelá-Ascoli theorem, and Lemma 10, we obtain the desired result.

5.3. Proof of Theorem 3. The proof is based on Schaefer fixed point theorem [17].

Lemma 11. Let $E$ be a Banach space. Assume that $T: E \rightarrow E$ is a continuous compact operator and the set

$$
V=\{u \in E: u=\lambda T u, 0 \leq \lambda \leq 1\}
$$

is bounded. Then $T$ has a fixed point in $E$.

Proof. The continuity and the compactness of the mapping $F: Z \rightarrow Z$ follow from Lemmas 7 and 8 . We have just to show that the set

$$
\begin{gathered}
V=\left\{(x, y) \in Z:(x, y)=\left(\lambda F_{1}(x, y), \lambda F_{2}(x, y)\right),\right. \\
0 \leq \lambda \leq 1\}
\end{gathered}
$$

is bounded with respect to the norm $\|\cdot\|_{Z}$. Then, the result will be obtained by applying Lemma 11 .

Let $(x, y) \in Z$ such that $x=\lambda F_{1}(x, y)$ and $y=\lambda F_{2}(x, y)$ for some $\lambda \in[0,1]$. For all $t \in[0,1]$, using (i), (iii), and Lemma 6 , we obtain that

$$
\begin{aligned}
& |x(t)| \\
& =\lambda\left|\int_{0}^{1} G(t, s) f(s, x(s), y(s)) d s+\frac{\xi(x(1))}{2} t\left(\frac{t^{2}}{3}-1\right)\right| \\
& \leq \frac{a}{2}+\frac{\epsilon}{2}\|x\|_{\infty}+\frac{\|x\|_{\infty}}{2},
\end{aligned}
$$

which implies that

$$
\|x\|_{\infty} \leq \frac{a}{1-\epsilon} .
$$

Similarly, using (ii), (iii), and Lemma 6 , for all $t \in[0,1]$, we have

$$
\begin{aligned}
& |y(t)| \\
& =\lambda\left|\int_{0}^{1} G(t, s) g(s, x(s), y(s)) d s+\frac{\zeta(y(1))}{2} t\left(\frac{t^{2}}{3}-1\right)\right| \\
& \leq \frac{b}{2}+\frac{\delta}{2}\|y\|_{\infty}+\frac{\|y\|_{\infty}}{2},
\end{aligned}
$$


which implies that

$$
\|y\|_{\infty} \leq \frac{b}{1-\delta} .
$$

Consequently, $V$ is a bounded set.

5.4. Proof of Theorem 4. The following fixed point theorem due to Agarwal et al. [18] will be used in the proof.

Lemma 12. Let $E$ be a Banach space, let $D$ be a bounded closed convex subset of $E$, and let $A, B: D \rightarrow E$ such that $A u+B v \in$ $D$ for every $(u, v) \in D$. If $A$ is a contraction and $B$ is continuous and compact, then the equation $A w+B w=w$ has a solution $w \in D$.

Proof. We define the operators $A, B: Z \rightarrow Z$ as follows: for all $(x, y) \in Z$,

$$
\begin{gathered}
A(x, y)=\left(A_{1}(x, y), A_{2}(x, y)\right), \\
B(x, y)=\left(B_{1}(x, y), B_{2}(x, y)\right),
\end{gathered}
$$

where, for every $t \in[0,1]$,

$$
\begin{gathered}
A_{1}(x, y)(t)=\frac{\xi(x(1))}{2} t\left(\frac{t^{3}}{3}-1\right), \\
A_{2}(x, y)(t)=\frac{\zeta(y(1))}{2} t\left(\frac{t^{3}}{3}-1\right), \\
B_{1}(x, y)(t)=\int_{0}^{1} G(t, s) f(s, x(s), y(s)) d s, \\
B_{2}(x, y)(t)=\int_{0}^{1} G(t, s) g(s, x(s), y(s)) d s .
\end{gathered}
$$

Clearly, for every $(x, y) \in Z$, we have

$$
F(x, y)=A(x, y)+B(x, y) .
$$

Let $2 \max \{a, b\}<R<1$. We define the set $D \subset Z$ as follows:

$$
D=\left\{(x, y) \in Z:\|(x, y)\|_{Z} \leq R\right\} .
$$

Let $(x, y),(u, v) \in D$. For every $t \in[0,1]$, we have

$$
\begin{aligned}
& \left|A_{1}(x, y)(t)+B_{1}(u, v)(t)\right| \\
& \quad \leq \int_{0}^{1} G(t, s)|f(s, u(s), v(s))| d s+\frac{|\xi(x(1))|}{2}
\end{aligned}
$$

(from Lemma 6 and conditions (i), (iii), and (iv))

$$
\begin{aligned}
& \leq \frac{1}{2}\left(a+\epsilon_{1} R^{\rho_{1}}+\epsilon_{2} R^{\rho_{2}}\right)+\frac{R}{2} \\
& \leq \frac{a+\left(\epsilon_{1}+\epsilon_{2}+1\right)}{2} R \\
& \leq \frac{1 / 2+\left(\epsilon_{1}+\epsilon_{2}+1\right)}{2} R \leq R .
\end{aligned}
$$

Similarly, for every $t \in[0,1]$, we have

$$
\begin{aligned}
& \left|A_{2}(x, y)(t)+B_{2}(u, v)(t)\right| \\
& \quad \leq \int_{0}^{1} G(t, s)|g(s, u(s), v(s))| d s+\frac{|\zeta(u(1))|}{2}
\end{aligned}
$$

(from Lemma 6 and conditions (ii), (iii), and (iv))

$$
\begin{aligned}
& \leq \frac{1}{2}\left(b+\delta_{1} R^{\sigma_{1}}+\delta_{2} R^{\sigma_{2}}\right)+\frac{R}{2} \\
& \leq \frac{b+\left(\delta_{1}+\delta_{2}+1\right)}{2} R \\
& \leq \frac{1 / 2+\left(\delta_{1}+\delta_{2}+1\right)}{2} R \leq R .
\end{aligned}
$$

Thus, we proved that, for every $(x, y),(u, v) \in D$, we have

$$
A(x, y)+B(u, v) \in D \text {. }
$$

Now, we will prove that $A: D \rightarrow Z$ is a contraction. Let $(x, y),(u, v) \in D$. For every $t \in[0,1]$, we have

$$
\begin{aligned}
\left|A_{1}(x, y)(t)-A_{1}(u, v)(t)\right| & \leq \frac{|\xi(x(1))-\xi(u(1))|}{2} \\
(\text { from condition (iii) }) & \leq \frac{k}{2}|x(1)-u(1)| \\
& \leq \frac{k}{2}\|x-u\|_{\infty} .
\end{aligned}
$$

Similarly, for every $t \in[0,1]$, we have

$$
\begin{aligned}
\left|A_{2}(x, y)(t)-A_{2}(u, v)(t)\right| & \leq \frac{|\zeta(y(1))-\zeta(v(1))|}{2} \\
(\text { from condition (iii)) } & \leq \frac{k}{2}|y(1)-v(1)| \\
& \leq \frac{k}{2}\|y-v\|_{\infty} .
\end{aligned}
$$

Since $0<k<2$, then $A$ is a contraction (with respect to the norm $\|\cdot\|_{Z}$ ). Finally, the continuity and the compactness of the operator $B$ follow by using the same arguments of the proof of Theorem 2. Applying Lemma 12, we obtain the existence of $(x, y) \in D$ such that $(x, y)$ is a fixed point of $A+B=F$.

\section{Acknowledgment}

The author would like to extend his sincere appreciation to the Deanship of Scientific Research at King Saud University for the funding of this research through the Research Group Project no. RGP-VPP-237.

\section{References}

[1] C. P. Gupta, "A nonlinear boundary-value problem associated with the static equilibrium of an elastic beam supported 
by sliding clamps," International Journal of Mathematics and Mathematical Sciences, vol. 12, no. 4, pp. 697-711, 1989.

[2] G. Bonanno and B. Di Bella, "A boundary value problem for fourth-order elastic beam equations," Journal of Mathematical Analysis and Applications, vol. 343, no. 2, pp. 1166-1176, 2008.

[3] C. J. Chyan and J. Henderson, "Positive solutions of $2 \mathrm{mth}$-order boundary value problems," Applied Mathematics Letters, vol. 15, no. 6, pp. 767-774, 2002.

[4] J. R. Graef and B. Yang, "On a nonlinear boundary value problem for fourth order equations," Journal of Applied Analysis, vol. 72, pp. 439-448, 1999.

[5] C. P. Gupta, "Existence and uniqueness theorems for the bending of an elastics beam equation," Journal of Applied Analysis, vol. 26, no. 4, pp. 289-304, 1988.

[6] P. Kang and L. Liu, "Positive solutions of fourth order singular boundary value problems," Journal of Systems Science and Mathematical Sciences, vol. 28, pp. 604-615, 2008 (Chinese).

[7] Y. Li, "On the existence of positive solutions for the bending elastic beam equations," Applied Mathematics and Computation, vol. 189, no. 1, pp. 821-827, 2007.

[8] Q. Yao, "Existence of $\mathrm{n}$ solutions and/or positive solutions to a semipositone elastic beam equation," Nonlinear Analysis: Theory, Methods and Applications, vol. 66, no. 1, pp. 138-150, 2007.

[9] R. P. Agarwal, "On fourth order boundary value problems arising in beam analysis," Differential Integral Equations, vol. 2, pp. 91-110, 1989.

[10] Z. Bai, "The method of lower and upper solutions for a bending of an elastic beam equation," Journal of Mathematical Analysis and Applications, vol. 248, no. 1, pp. 195-202, 2000.

[11] M. A. del Pino and R. F. Manasevich, "Existence for a fourthorder boundary value problem under a two parameter nonresonance condition," Proceedings of the American Mathematical Society, vol. 112, pp. 81-86, 1991.

[12] D. R. Dunninger, "Multiplicity of positive solutions for a nonlinear fourth order equation," Annales Polonici Mathematici, vol. 77, no. 2, pp. 161-168, 2001.

[13] D. O'Regan, “Solvability of some fourth (and higher) order singular boundary value problems," Journal of Mathematical Analysis and Applications, vol. 161, no. 1, pp. 78-116, 1991.

[14] P. Kang and Z. Wei, "Existence of positive solutions for systems of bending elastic beam equations," Electronic Journal of Differential Equations, vol. 2012, pp. 1-9, 2012.

[15] A. I. Perov, "On the Cauchy problemfor a system of ordinary differential equations," Pviblizhen. Met. Reshen. Differ. Uvavn, vol. 2, pp. 115-134, 1964.

[16] D. A. Gilbarg and N. S. Trudinger, Elliptic Partial Differential Equations of Second Order, vol. 224, Springer, Berlin, Germany, 2001.

[17] J. X. Sun, Nonlinear Functional Analysis and Its Application, Science Press, Beijing, China, 2008.

[18] R. P. Agarwal, Y. Zhou, and Y. He, "Existence of fractional neutral functional differential equations," Computers and Mathematics with Applications, vol. 59, no. 3, pp. 1095-1100, 2010. 


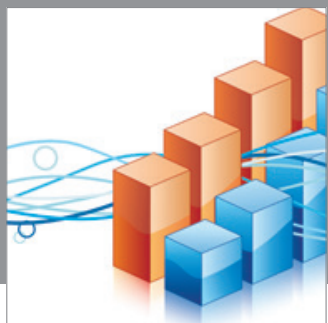

Advances in

Operations Research

mansans

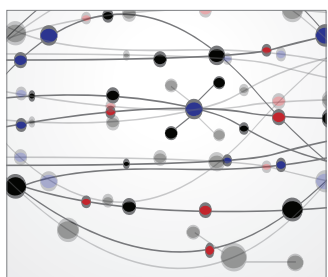

The Scientific World Journal
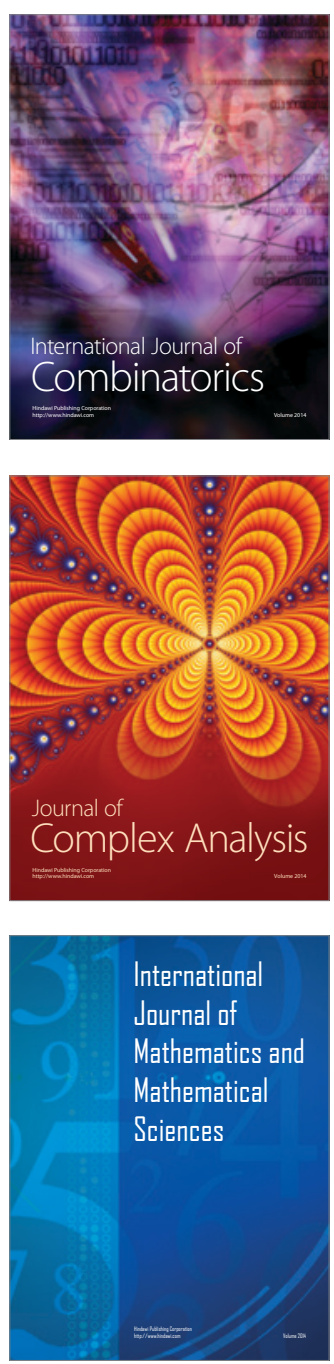
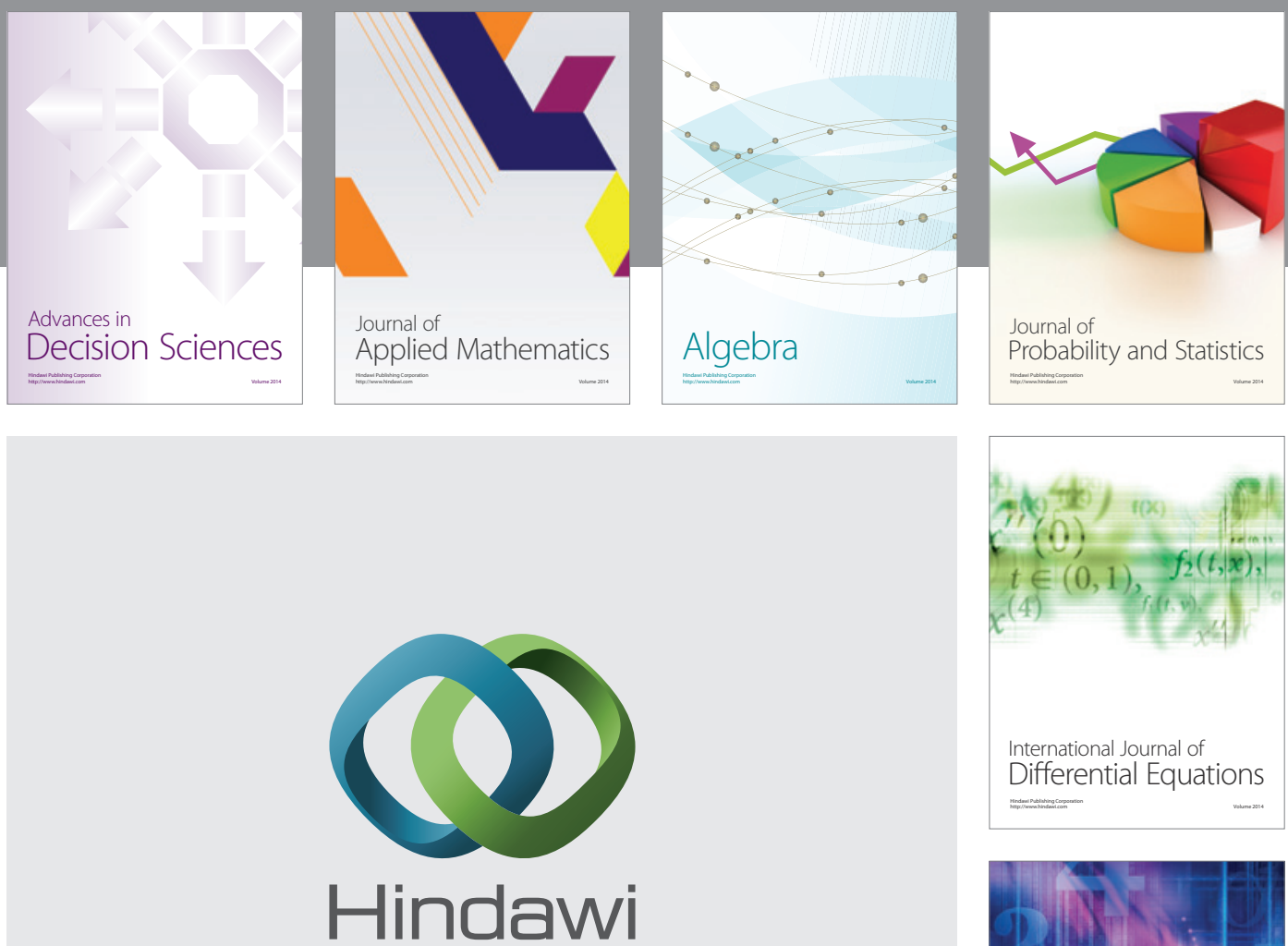

Submit your manuscripts at http://www.hindawi.com
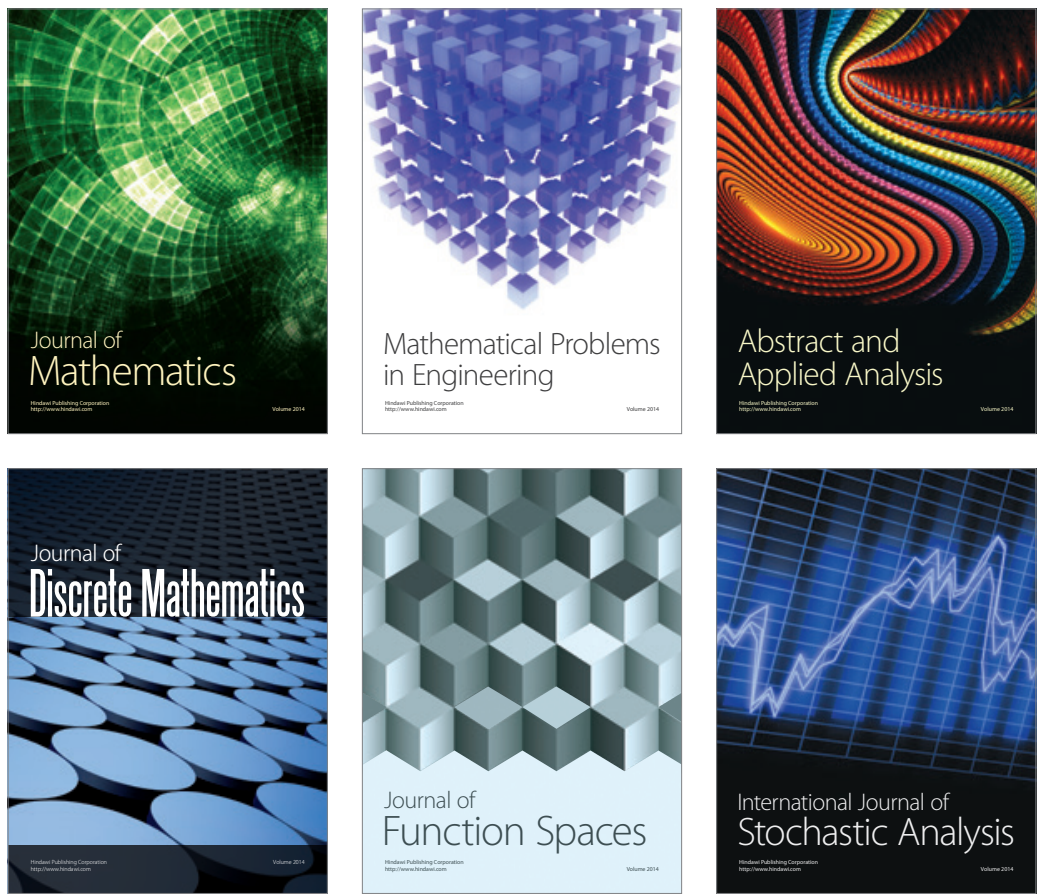

Journal of

Function Spaces

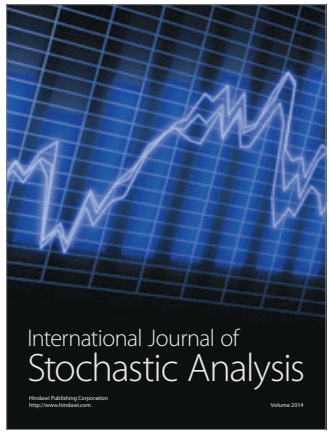

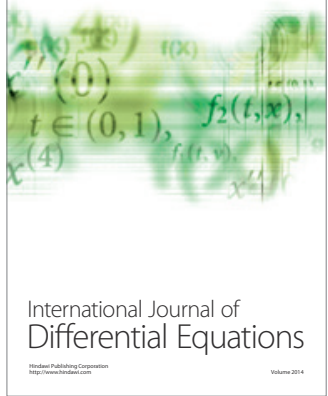
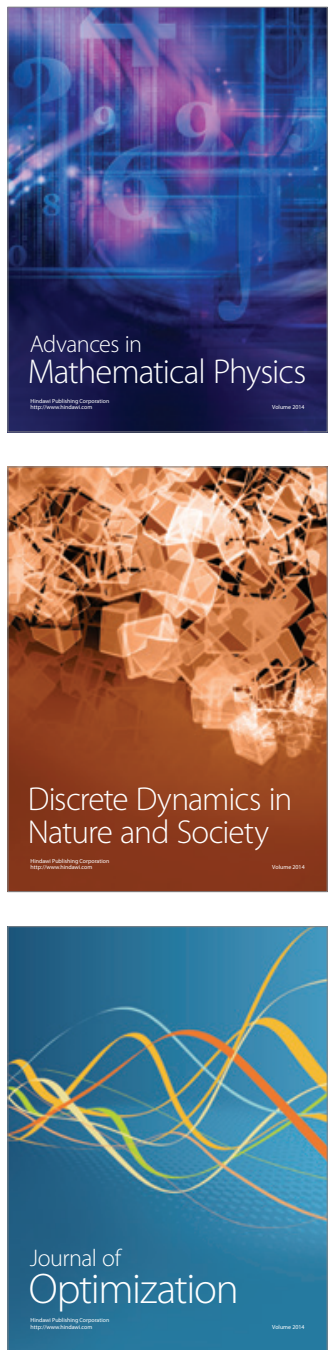